\title{
Reproducibility of late gadolidium enhancement of atrial ablation scar
}

\author{
Henry Chubb ${ }^{1 *}$, Rashid Karim ${ }^{1}$, John Whitaker ${ }^{1}$, James Harrison ${ }^{1}$, Matthew Wright ${ }^{1,2}$, Mark O'Neill ${ }^{1,2}$, Reza Razavi ${ }^{1}$ \\ From 19th Annual SCMR Scientific Sessions \\ Los Angeles, CA, USA. 27-30 January 2016
}

\section{Background}

Late gadolinium enhancement (LGE) can be used to visualise post-ablation scar in the left atrium, such as following pulmonary isolation procedures for atrial fibrillation. Studies have demonstrated a correlation between ablation site, LGE scar formation and clinical outcomes. However, the reproducibility of scar imaging is undetermined.

\section{Methods}

Eleven patients with atrial fibrillation (AF, 4 persistent) underwent first time pulmonary vein isolation procedure. 3D LGE magnetic resonance imaging (1.5T Philips Ingenia) was performed at 3 months (91 \pm 15 days) post ablation (scan 1), with a reproducibility scan three days later (scan 2). 3D LGE acquisitions were performed at 10,20 and $30 \mathrm{~min}$ post gadolinium (Gd, $0.2 \mathrm{mmol} / \mathrm{kg}$ ) (spatial resolution $1.3 \times 1.3 \times 4 \mathrm{~mm}$, reconstructed to $0.6 \times 0.6 \times 2 \mathrm{~mm}$ ). Scan 2 was performed $48-96$ hours after the Scan 1 .

The left atrium (LA) was manually segmented and LGE enhancement projected onto the shell (maximum intensity projection technique). Each pulmonary vein (PV) was analysed by 4 independent observers. The percentage of scar encirclement of each PV was quantified visually between $0 \%$ (no scar) and $100 \%$ (complete encirclement of vein by scar). The LA shell was thresholded at 3.3 standard deviations above the blood pool mean.

\section{Results}

Sixty six 3D LGE sequences were acquired, totalling 1056 PV measurements (Figure 1). There was good agreement between observers (ICC 0.885 (95\% CI 0.85-0.91)). There was significantly less enhancement surrounding the

${ }^{1}$ Institute of Imaging Sciences and Biomedical Engineering, King's College London, London, United Kingdom

Full list of author information is available at the end of the article
LUPV compared to other veins ( $\mathrm{p}<0.001$, KruskallWallis), and observed PV encirclement was significantly lower at $10 \mathrm{~min}$ post $\mathrm{Gd}$ than at 20 or $30 \mathrm{~min}(21 \pm 28 \%$ vs $69 \pm 28 \%, \mathrm{p}<0.001)$. Imaging performed $10 \mathrm{~min}$ post Gd was therefore not assessed for reproducibility, as it was judged an insensitive measure of scar imaging.

Pulmonary vein encirclement was moderately reproducible. On assessment of maximum encirclement of each individual vein (the higher figure for each vein at either $20 \mathrm{~min}$ or $30 \mathrm{~min}$ post Gd), mean error between Scan 1 and Scan 2 was $2.0( \pm 10.9) \%$. For mean encirclement, averaging measurements for each vein at $20 \mathrm{~min}$ and 30 min, mean error was $0.7 \%( \pm 18.4 \%)$.

\section{Conclusions}

The utility of LGE assessment of left atrial scar following ablation in guiding further interventions has varied between centres, and a study of the reproducibility of LGE assessment of atrial scar is clearly required. This study suggests that there is a good degree of reproducibility, but that it is highly dependent upon the timing of the acquisition following gadolinium administration. Current data suggests that the main shortfall of LGE scar assessment is one of sensitivity of scar detection, rather than specificity. An approach of multiple acquisitions, and assessment of maximum scar, may be the most reproducible.

\section{Authors' details \\ 'Institute of Imaging Sciences and Biomedical Engineering, King's College London, London, United Kingdom. ${ }^{2}$ Cardiology, Guy's and St Thomas' NHS Foundation Trust, London, United Kingdom.}

Published: 27 January 2016

doi:10.1186/1532-429X-18-S1-Q28

Cite this article as: Chubb et al: Reproducibility of late gadolidium enhancement of atrial ablation scar. Journal of Cardiovascular Magnetic Resonance 2016 18(Suppl 1):Q28. 


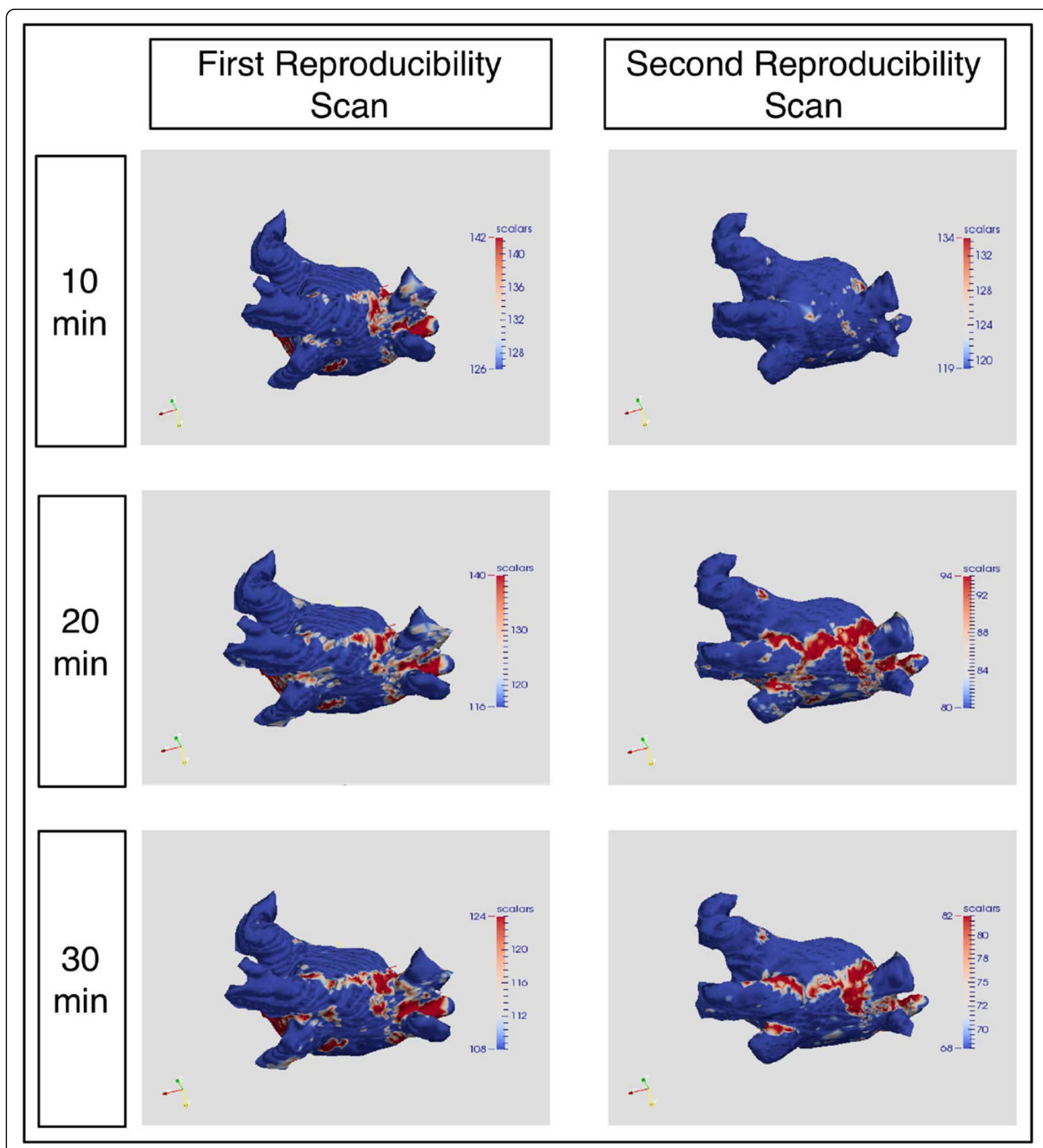

Figure 1 Left atrial shells generated from 3D LGE acquisition. Signal intensity derived from maximum intensity projection interogation and shells thresholded at 3.3SD above the blood pool mean. 